\title{
Chlamydia trachomatis Antigen Measurement
}

National Cancer Institute

\section{Source}

National Cancer Institute. Chlamydia trachomatis Antigen Measurement. NCI Thesaurus.

Code C154835.

The determination of the Chlamydia trachomatis antigen present in a sample. 